\title{
Politique cotonnière en Syrie : adaptation partielle et progressive à la mondialisation
}

\author{
Haitham Alashkar ${ }^{1}$ \\ Michel Fok ${ }^{2}$ \\ ${ }^{1}$ National Agricultural Policy Centre \\ Ministry of Agriculture \\ Airport Highway \\ Fifth Bridge \\ PO Box 4251 \\ Damascus \\ Syrian Arab Republic \\ <haitham.alashkar@napcsyr.org> \\ ${ }^{2}$ Cirad \\ Avenue Agropolis \\ TA B102/02 \\ 34398 Montpellier \\ France \\ <michel.fok@cirad.fr>
}

\begin{abstract}
Résumé
La Syrie est un pays producteur de coton, significatif, mais sa politique cotonnière est rarement analysée et reste largement méconnue. C'est une politique assez particulière, qui se caractérise par l'engagement fort et stable de l'État et par la puissance du contrôle administratif de la production, même après une certaine libéralisation de l'économie pour s'adapter à la mondialisation. Le système de prix, inchangé depuis plus de quarante ans, a garanti des revenus attractifs aux producteurs. La Syrie fournit un rare exemple de politique publique fondée sur le contrôle de l'offre nationale de coton, intégrant récemment le souci de durabilité dans l'usage de la ressource en eau. Au regard des objectifs retenus, la politique conduite s'est révélée efficace pour augmenter la production et pour développer une industrie textile dont les besoins en matière première sont largement couverts par la production nationale. Les investissements en recherche et en appui technique ont permis par ailleurs à la Syrie d'être l'un des rares pays à réduire fortement le recours aux insecticides. La difficulté d'accès aux données ne permet cependant pas d'évaluer précisément l'efficacité de la politique conduite. La prise en compte d'une gestion durable de l'eau semble par exemple encore bien insuffisante. Le souhait de ce pays d'adhérer à l'Organisation mondiale du commerce (OMC) lui impose le défi d'une refonte de sa politique cotonnière tout en préservant un rôle efficace de l'État.
\end{abstract}

Mots clés : coton ; irrigation ; politique agricole ; politique de soutien ; Syrie.

Thèmes : eau ; économie et développement rural ; productions végétales.

\section{Abstract \\ Cotton policy in Syria: Partial and gradual adaptation to globalization}

Syria is a rather important cotton producing country but its cotton policy is seldom appraised and remains little known outside the country. This policy is quite particular given a strong and stable commitment from the State and powerful administrative control of cotton production, even after some shift to a more liberalized economy. The pricing system has remained unchanged for more than forty years to guaranty producers an attractive income. Syria provides a rare example of a cotton policy based on a controlled offer, integrating more recently the concern of sustainable water use. With regard to the policy objectives, the Syrian cotton policy is proving effective at increasing cotton production while developing a textile industry the raw material requirements of which are fully covered. The investment in research and technical assistance to producers has enabled Syria to be a rare cotton country achieving substantial reduction of insecticide use. It is not possible to assess the extent of policy effectiveness and efficiency because of difficult access to the needed data. However, concern for a sustainable use of water appears quite insufficient. The Syrian desire to join the WTO implies the challenge of drastic change of its cotton policy while maintaining an effective role of the State.

Key words: agricultural policies; cotton; irrigation; support measures; Syria.

Subjects: economy and rural development; vegetal productions; water.

Tirés à part : M. Fok 
a production de coton existe depuis longtemps en Syrie mais son développement remonte à un demisiècle, avec notamment la nationalisation du secteur coton en 1965 (Macrae, 1995). La singularité du contrôle de la production, par la délivrance de permis de culture, constitue un cas extrême d'intervention de l'État dans le monde du coton. Cette forte emprise de l'État donne lieu à des appréciations négatives (Westlake, 2003) qui occultent cependant le fait que la Syrie est le seul pays à gérer sa production cotonnière en fonction de la ressource en eau et à réduire très fortement le recours aux pesticides. L'analyse du cas syrien participe à la capitalisation des modalités d'intervention des États dont le rôle régulateur est maintenant reconnu (World Bank, 2008), dans le but d'aider à adapter cette intervention à la diversité des modes d'organisation des filières cotonnières (Tschirley et al., 2009) et pour engager les États dans des arrangements institutionnels efficaces.

L'objectif de cet article est de restituer l'évolution de la politique cotonnière de la Syrie et d'en discuter l'efficacité. L'analyse est conduite dans la limite du faible accès aux données disponibles en raison du contexte politique du pays. La première partie de cet article évoque la contrainte des facteurs naturels. La deuxième partie décrit la politique cotonnière en soulignant sa stabilité et la force du contrôle par l'État. La troisième partie discute de son efficacité.

\section{Des facteurs naturels} contraignants

\section{Agriculture et coton dans l'économie syrienne}

L'agriculture est d'une grande importance économique en Syrie. En 2005, elle représentait $26 \%$ du produit intérieur brut (PIB). Le coton représentait $61 \%$ de la valeur totale des cultures industrielles et $15 \%$ de la valeur totale des exportations agricoles.

La production et la transformation du coton concernent un grand nombre de personnes, estimé à $20 \%$ de la population active (Shweih, 2006). Le coton fait partie des cultures stratégiques depuis plusieurs décennies (de même que le blé, l'orge, les lentilles, le pois chiche, la betterave et le tabac). A ce titre, le gouvernement n'a eu de cesse d'en promouvoir la production.

\section{Manque de terres et exigence en irrigation}

La Syrie dispose d'un territoire de 18,5 millions d'hectares, dont $32 \%$ seulement sont cultivables et $30 \%$ de la part cultivable sont irrigués aujourd'hui. Les exploitations agricoles sont de petite taille, $75 \%$ des exploitations ayant moins de 10 hectares, avec une moyenne de 2,87 hectares. Les zones où le coton est davantage cultivé ont des exploitations de taille nettement plus grande, soit 25 hectares en moyenne (Anonyme, 1995).

Sur la majeure partie des terres cultivées, la production est soumise aux faibles précipitations d'un pays semi-aride. Une division en cinq zones agroécologiques a été établie selon un gradient décroissant de pluviométrie et d'aptitude à la culture pluviale (figure 1). À titre indicatif, la première zone a une pluviosité annuelle supérieure à $350 \mathrm{~mm}$, mais la culture strictement pluviale n'est réellement sûre que là où elle est supérieure à $600 \mathrm{~mm}$. Au cours des années 1970, le gouvernement a largement investi dans la construction de barrages pour l'irrigation des cultures. En conséquence, grâce à l'irrigation, le coton est cultivé dans les cinq zones.

\section{Pression accentuée de l'irrigation sur une eau rare}

La Syrie dispose de faibles disponibilités en eau renouvelable. Elle est en moyenne de $361 \mathrm{~m}^{3} /$ habitant/an pour la période 2003-2007 (contre $2910 \mathrm{~m}^{3}$ en France pour la même période). La croissance démographique (2,45\% par an) contribue à réduire cette disponibilité qui a chuté de $65 \%$ par rapport à la période 1968-1972, contre $16 \%$ en France (FAO, 2009). L'eau disponible provient à $80 \%$ des eaux de surface et des eaux souterraines ; elle peut être fortement réduite en année sèche (cas de l'année 2000).

L'extension de l'irrigation fait que l'agriculture utilise $79 \%$ de l'eau consommée en 2001-2002. Ce taux peut approcher les $95 \%$ dans les zones où la production agricole est totalement dépendante de l'irrigation (tableau 1).

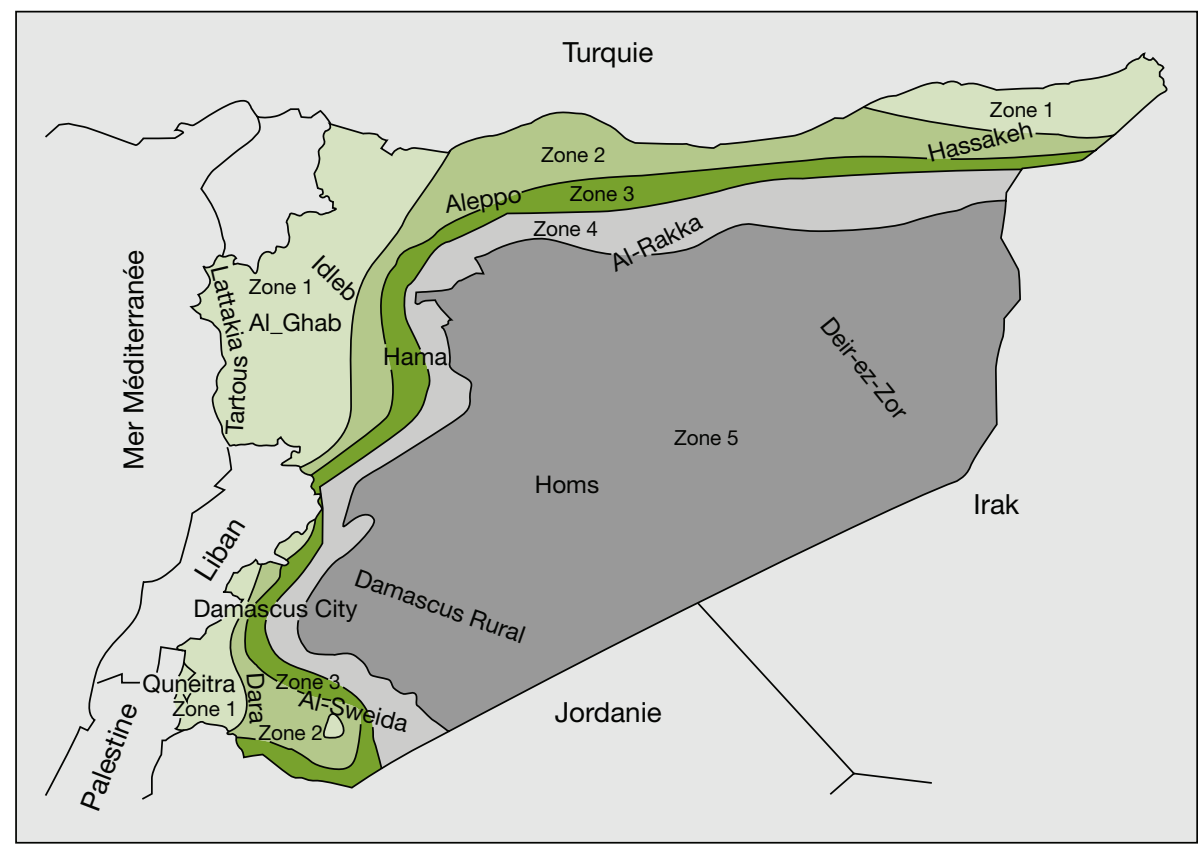

Figure 1. Zonage agroécologique de la Syrie.

Figure 1. Agroecological zoning of Syria.

Source : ministère de l'Agriculture et de la Réforme agraire. 
Tableau 1. Répartition des usages de l'eau en 2001-2002 en fonction des bassins hydrauliques.

Table 1. Distribution of water use in 2001-2002 according to watershed basins.

\begin{tabular}{|c|c|c|c|c|c|c|}
\hline \multirow[t]{2}{*}{ Bassin } & \multicolumn{5}{|c|}{ Eau utilisée par million de mètres cubes } & \multirow{2}{*}{$\begin{array}{c}\text { Part de } \\
\text { I'agriculture } \\
(\%)\end{array}$} \\
\hline & Agriculture & Industrie & Ménages & Évaporation & Total usage & \\
\hline Barada \&AI-Aawaj & 578 & 76 & 269 & 6 & 929 & 62,2 \\
\hline Al-Yarmook & 360 & 38 & 76 & 31 & 505 & 71,3 \\
\hline Al-Badia & 984 & 2 & 44 & 15 & 1045 & 94,2 \\
\hline Al-Aassi & 1735 & 229 & 240 & 148 & 2352 & 73,8 \\
\hline Zone côtière & 459 & 85 & 81 & 16 & 641 & 71,6 \\
\hline Al-Khabour & 4018 & 45 & 38 & 132 & 4233 & 94,9 \\
\hline Euphrates & 5498 & 86 & 322 & 1614 & 7520 & 73,1 \\
\hline Total & 13631 & 561 & 1070 & 1962 & 17224 & 79,1 \\
\hline
\end{tabular}

Source : ministère de l'Alimentation et de l'Agriculture.

\section{Des systèmes d'irrigation peu économes}

En Syrie, le système gravitaire reste le mode d'irrigation largement dominant; il concerne près de $85 \%$ des surfaces irriguées (tableau 2). L'eau est distribuée à la raie à travers des réseaux d'irrigation établis à partir des barrages (24\% des surfaces irriguées). Elle est aussi souvent pompée à partir de puits créés par les paysans eux-mêmes - c'est le cas de plus de 60 \% des surfaces irriguées ; enfin, le pompage dans les cours d'eau sans aménagement public représente tout de même plus de $15 \%$ des surfaces irriguées.

L'aspersion est considérée en Syrie comme une méthode moderne d'irrigation, au même titre que les dispositifs d'irrigation au goutte-à-goutte. Ce dernier système est encore faiblement utilisé : il ne représente que $6 \%$ des surfaces irriguées en 2006.

L'irrigation par pompage dans les puits, mode largement répandu, est peu efficace et concourt à épuiser les réserves d'eau souterraine. Une baisse du niveau de la nappe phréatique, de l'ordre de 10 à 20 mètres sur une période de 10 ans, est observée dans les zones irriguées par pompage dans les puits (Alabdullah et Nuppenau, 2006). La consommation en eau peut atteindre $500 \%$ de l'eau renouvelable dans certains bassins hydrauliques. Ces phénomènes témoignent de la raréfaction et d'un usage non durable de la ressource en eau; ils imposent la nécessité d'améliorer l'efficience de l'usage de l'eau en agriculture.

\section{Évolution lente}

\section{et originale} d'un fort contrôle de la production

Dans les pays producteurs de coton, l'intervention de l'État est assez fréquente. En Syrie, le contrôle par l'État passe par une planification administrative extrêmement poussée.

Il y a une grande stabilité de la politique cotonnière en Syrie depuis les années 1960 : ce qui a été décrit au début des années 1990 (Macrae, 1995) demeure en grande partie d'actualité alors que cela correspondait déjà à ce qui était présenté au début des années 1980 (Khoury, 1988)

\section{Tableau 2. Évolution des modes d'irrigation.}

Table 2. Evolution of irrigation modes.

\begin{tabular}{|c|c|c|c|c|c|c|c|c|}
\hline \multicolumn{5}{|c|}{$\begin{array}{l}\text { Surfaces irriguées } \\
\text { (1 } 000 \text { hectares) }\end{array}$} & \multicolumn{3}{|c|}{$\begin{array}{l}\text { Surfaces à irrigation moderne } \\
\text { (1 } 000 \text { hectares) }\end{array}$} & \multirow{2}{*}{$\begin{array}{c}\text { Part de } \\
\text { l'irrigation } \\
\text { moderne } \\
(\% \\
\text { surface totale) }\end{array}$} \\
\hline & Total & $\begin{array}{l}\text { Irrigation } \\
\text { par puits }\end{array}$ & $\begin{array}{c}\text { Réseaux } \\
\text { publics } \\
\text { d'irrigation }\end{array}$ & $\begin{array}{c}\text { Irrigation } \\
\text { par rivières } \\
\text { et cours d'eau }\end{array}$ & Sprinkler & $\begin{array}{l}\text { Goutte- } \\
\text { à-goutte }\end{array}$ & Total & \\
\hline 2002 & 1333 & 817 & 314 & 201 & 139 & 76 & 215 & 16,1 \\
\hline 2003 & 1361 & 855 & 289 & 217 & 133 & 52 & 185 & 13,6 \\
\hline 2004 & 1439 & 865 & 340 & 234 & 130 & 58 & 188 & 13,0 \\
\hline 2005 & 1426 & 865 & 326 & 234 & 160 & 84 & 244 & 17,1 \\
\hline 2006 & 1402 & 851 & 336 & 215 & 163 & 73 & 236 & 16,8 \\
\hline
\end{tabular}

Source : ministère de l'Alimentation et de l'Agriculture. 
ou 1970 (Anonyme, 1972). Pour autant, dans sa démarche d'une évolution progressive vers la libéralisation économique, on observe que la Syrie met en œeuvre des modalités originales d'administration de la production cotonnière qui ne sont pas dénuées d'efficacité.

\section{Contrôle par la planification administrative}

La production agricole en Syrie reste largement planifiée, surtout pour les productions jugées stratégiques telles que le coton. Cette planification procède de la définition d'une stratégie décennale qui est mise en œuvre dans le cadre de plans quinquennaux. Chaque année, la production cotonnière est planifiée jusqu'au niveau des villages.

Parmi les objectifs de la stratégie actuelle de développement de l'agriculture (couvrant la période 2001-2010), le gouvernement syrien a retenu d'étendre les superficies sous irrigation tout en en améliorant l'efficience par le recours aux méthodes modernes. A moyen terme, il est prévu d'atteindre ces objectifs en ajustant les modalités d'appui à l'agriculture pour les rendre compatibles avec les règles de l'Organisation mondiale du commerce (OMC) à laquelle la Syrie a fait acte de candidature en 2003. Les intrants sont ainsi fournis aujourd'hui aux paysans aux prix réels par les services du gouvernement. Dans le domaine particulier du coton, le système de crédit a été amendé pour intégrer des incitations financières à la conversion aux nouvelles techniques d'irrigation.

La planification annuelle débute en avril au niveau du ministère de l'Agriculture et de la Réforme agraire (Mara) pour fixer la surface annuelle et sa répartition entre les gouvernorats. La répartition au niveau de chaque échelon administratif, depuis le gouvernorat jusqu'au village, est réalisée en tenant compte de la disponibilité de l'eau pour irrigation (Westlake, 2001).

Chaque paysan doit obtenir un permis de culture du coton auprès des services agricoles. La Syrie est ainsi le seul pays cotonnier à contrôler la production de cette manière. À la délivrance des permis, des copies sont communiquées à la Banque des coopératives agricoles (BCA) qui est chargée de la fourniture des intrants et des crédits aux paysans.

\section{Contrôle par la fourniture des intrants et services à la production}

Comme dans beaucoup de pays où la production de coton n'est pas libéralisée, l'État syrien assume le rôle de fourniture des intrants et des services d'appui aux producteurs. Les modalités suivies ne sont pas très originales, à ceci près que c'est le permis de culture qui ouvre l'accès aux intrants et services.

Les semences produites par un organisme public (General Establishment for Seed Multiplications) sont vendues à la BCA qui les cède aux producteurs de coton. La Syrie applique rigoureusement le principe d'une seule variété par région. Le prix des semences (non délintées) a été fixé depuis 1996 à $10 \mathrm{LS}^{1} / \mathrm{kg}$ (soit 0,2 dollar US) et il n'a pas évolué depuis.

Les doses d'engrais distribuées sont celles recommandées par la recherche. Les sacs distribués pour la récolte sont en jute, avec des prix différenciés en fonction de leur état neuf ou usagé. Tous les intrants agricoles sont fournis à crédit mais aux prix réels depuis 1992. Le crédit est déduit des sommes perçues par les agriculteurs lors de la commercialisation du cotongraine.

En plus du crédit en nature lors de la fourniture des intrants, la BCA octroie aux producteurs une avance de trésorerie à raison de 5000 LS par hectare (soit 100 dollars US/ha). Les taux d'intérêt sont différenciés en fonction de la nature des bénéficiaires : $5 \%$ pour les fermes d'État, $7 \%$ pour les coopératives agricoles et $8 \%$ pour les producteurs individuels, mais ils sont bien inférieurs aux 30-40\% des opérateurs privés.

Les paysans bénéficient de la subvention pour les carburants et l'électricité comme tout citoyen syrien. Jusqu'à 2007-2008, les taux de subvention étaient respectivement de $70 \%$ du prix mondial et $30 \%$ du coût réel (Al-Joumani, 2004).

La recherche cotonnière est publique et centralisée comme dans beaucoup de pays cotonniers. Depuis 2001, tous les organismes de recherche sont regroupés au sein d'une institution unique, la Commission for Scientific Agricultural Research (GCSAR). La recherche sur le coton est sous la responsabilité du Cotton Bureau de la GCSAR.

\footnotetext{
${ }^{1}$ LS : livre syrienne. Taux de change 2008: 1 euro $=72,89$ LS.
}

Un programme spécifique du Mara fournit l'appui technique aux paysans. Le personnel d'encadrement technique dispose d'un pouvoir très fort pour imposer le respect des techniques culturales par les paysans. Cela s'applique en particulier au domaine du contrôle contre les ravageurs du cotonnier. Depuis 1988, la Syrie a entrepris de favoriser la lutte intégrée contre les ravageurs du cotonnier afin de réduire le recours aux insecticides. C'est le seul pays où l'État apporte un soutien à la production de coton biologique. En pratique, les producteurs ne peuvent obtenir aujourd'hui les produits insecticides ni ne peuvent procéder aux traitements sans le consentement du personnel d'encadrement technique.

\section{Contrôle du commerce des produits du coton}

\section{Une commercialisation \\ fortement centralisée}

Tous les producteurs ont l'obligation de vendre toute leur récolte de coton à la General Organization for Cotton Ginning and Marketing (ou CMO) relevant du ministère de l'industrie, et d'ailleurs seulement de 3 à $5 \%$ de la production y échappe (Macrae, 1995 ; Shweih, 2006).

L'achat aux producteurs est réalisé au prix déterminé par la CMO. Le prix d'achat du coton-graine est calculé pour couvrir le coût total de production et pour donner une marge de profit de $15 \%$. Le travail familial est pris en compte dans le coût total de production. Depuis la campagne 1996-1997, les différentiels de prix sont établis pour encourager les récoltes et livraisons précoces :

$-30,75 \mathrm{LS} / \mathrm{kg}$, en début de saison jusqu'au 15 novembre;

- 26,25 LS/kg du 16 au 30 novembre ;

- 19,75 LS/kg à partir de décembre.

Ces prix n'ont pas évolué jusqu'à une date récente (Macrae, 1995; Shweih, 2006) : c'est seulement en 2008 que le premier prix (pour la récolte précoce) a été relevé et porté à $41 \mathrm{LS} / \mathrm{kg}$.

En plus de la période de livraison, comme dans la plupart des pays cotonniers, les caractéristiques de qualité de fibre (mesurées sur échantillon venant d'un égrenage à rouleau à la livraison) sont prises en compte pour déterminer le prix payé aux producteurs. Les prix indiqués cidessus concernent le coton ayant les meilleures caractéristiques de qualité. 
Échanges mondiaux et approvisionnement de l'industrie textile syrienne

La CMO est le seul organisme habilité à transformer le coton-graine dans ses usines d'égrenage et à vendre le coton-fibre. La vente du coton-fibre doit d'abord satisfaire les besoins de l'industrie textile nationale.

Jusqu'à 2001, le coton-fibre était fourni aux filateurs locaux au coût réel (en tenant compte du prix payé aux producteurs), plus une marge de $15 \%$. En d'autres termes, les filateurs étaient taxés pour prendre en charge le prix de soutien alloué aux paysans, au moins pour les quantités qu'ils transformaient.

\section{Évolution du contrôle par l'État}

À partir du début des années 2000, la Syrie a opté pour une libéralisation progressive de son économie agricole. Dans le domaine de la production cotonnière, cette évolution se caractérise par la mise en ouvre d'une gestion tenant compte de la disponibilité en eau ainsi qu'un approvisionnement plus favorable de l'industrie textile.

Une planification de la production en rapport avec les ressources en eau

Depuis 2002, la planification des superficies donnant lieu à permis de culture tient compte des disponibilités locales en eau. La culture du coton sans permis est devenue possible mais elle ne donne droit ni au prix de soutien à l'achat du coton- graine ni à la fourniture des intrants par la BCA. Le coton qui en est issu est aussi commercialisé par la $\mathrm{CMO}$, mais au prix mondial, bien moins favorable. En 2002, le prix mondial appliqué au coton cultivé sans permis était de 14,5 LS/kg (ou 0,28 dollar US/kg), soit moins de la moitié du prix garanti pour du coton cultivé avec permis et livré précocement.

La Syrie est ainsi le seul pays cotonnier à appliquer une politique de soutien qui ne couvre pas l'ensemble de la production. Les agents du Mara sont responsables de la délivrance des certificats d'origine après la récolte des paysans, ce qui permet ainsi de distinguer les productions venant des surfaces avec ou sans permis.

La prise en compte de la baisse des ressources en eau a conduit le gouvernement syrien à restreindre davantage la culture du coton aux zones où cette ressource reste suffisante. Globalement, cela s'est traduit par une diminution des surfaces planifiées (tableau 3), mais elle est d'ampleur limitée. Pour autant, c'est le seul exemple au monde de gestion étatique de la production cotonnière en relation avec la ressource en eau.

L'autorisation de la culture sans permis, dans les zones où la production cotonnière n'est pas désirée par le gouvernement, révèle l'amorce d'une gestion de l'offre par les signaux de marché. Le gouvernement syrien semble tabler sur la faible attractivité des prix de marché pour que les paysans des zones concernées arrêtent d'eux-mêmes la production cotonnière.
Une évolution prudente dans la politique d'irrigation

Alors que le prix garanti est le même sur tout le territoire syrien, les coûts de production, notamment ceux liés à l'irrigation ne sont pas les mêmes pour tous les paysans. Lorsque l'irrigation est réalisée à partir du dispositif public de canaux, les paysans paient seulement une redevance pour participer à l'entretien du réseau, indépendante de la quantité d'eau consommée. Avec l'irrigation par pompage dans les puits, les paysans ont la charge des frais d'exhaure (pompe, carburant, maintenance) qui sont bien supérieurs à la redevance dans le cas précédent (Alabdullah et Nuppenau, 2006) même si l'eau reste aussi gratuite.

Pour éviter que les paysans irriguant par pompage dans les puits n'abandonnent la culture de coton, alors qu'ils sont fort nombreux, le gouvernement syrien leur octroie une subvention directe de $30000 \mathrm{LS} /$ ha. Ce faisant, il entretient un mode d'irrigation peu efficace et peu économe en eau.

Pour réduire ces gaspillages, le gouvernement vise à faire évoluer les techniques d'irrigation. Dans le plan quinquennal en cours, il a établi un fonds spécial (1,1 milliard de dollars US) pour octroyer des prêts d'équipement à long terme sans intérêt. Par ailleurs, un abattement du capital à rembourser est appliqué, de 10 à $40 \%$ en fonction du mode initial d'irrigation. Cet abattement est plus important pour les paysans irriguant par pompage dans les puits.

\section{Tableau 3. Évolution des surfaces totales et à permis de coton.}

Table 3. Evolution of total and licensed cotton areas.

\begin{tabular}{lcccc}
\hline & $\begin{array}{c}\text { Surfaces planifiées } \\
\text { avec permis } \\
\text { (hectares) }\end{array}$ & $\begin{array}{c}\text { Surfaces } \\
\text { sans permis } \\
\text { (hectares) }\end{array}$ & $\begin{array}{c}\text { Surfaces totales réalisées } \\
\text { (hectares) }\end{array}$ & $\begin{array}{c}\text { Part des surfaces } \\
\text { sans permis } \\
\text { (\%) }\end{array}$ \\
\hline 1997 & 230000 & 20600 & 250600 & 8,2 \\
1998 & 240080 & 34505 & 274585 & 12,6 \\
1999 & 255200 & 0 & 243835 & 0,0 \\
2000 & 236000 & 34290 & 270290 & 12,7 \\
2001 & 205933 & 51130 & 257063 & 19,9 \\
2002 & 180600 & 19173 & 199773 & 9,6 \\
2003 & 206069 & 0 & 205360 & 0,0 \\
2004 & 215755 & 18426 & 234181 & 7,9 \\
2005 & 221583 & 16185 & 237768 & 6,8 \\
2006 & 221583 & 0 & 215640 & 0,0 \\
2007 & 209834 & 0 & 192790 & 0,0
\end{tabular}

Source : ministère de l'Agriculture et de la Réforme agraire. 
Une amélioration

des modalités d'approvisionnement de I'industrie textile

Une telle amélioration reste toute relative. Pour forcer son industrie textile à s'approvisionner dans le pays, la Syrie est le seul pays à maintenir l'interdiction totale de l'importation de coton-fibre alors que d'autres pays (comme les États-Unis, la Chine ou l'Inde) usent de quotas ou de taxes élevées à l'importation.

Depuis 2001, les conditions d'approvisionnement local sont devenues moins pénalisantes pour l'industrie textile. Les filateurs paient le coton-fibre au prix mondial, en monnaie locale, après conversion suivant le taux de change du marché. En cessant de taxer cette industrie, il est attendu un gain de compétitivité qui augmenterait la demande en coton local.

L'ouverture du secteur de la filature aux opérateurs privés reste faible. Les entreprises publiques restent très largement dominantes et elles sont favorisées dans leur approvisionnement en coton car elles bénéficient d'une remise de 8 cents US $/ \mathrm{kg}$.

\section{Efficacité et efficience de la politique} mise en œuvre

D'un point de vue libéral, le fait que le coton soit exporté à perte, peut amener à considérer comme inefficace la politique cotonnière en Syrie. Une telle vision est discutable puisque l'ensemble des objectifs fixés par l'État est atteint. Pour autant, les effets négatifs d'une politique d'encouragement à l'exploitation de l'eau souterraine indiquent la nécessité de faire évoluer encore plus la politique cotonnière.

\section{Exportation à perte : signe d'inefficacité ?}

L'appréciation de l'efficacité de la politique cotonnière en Syrie a donné lieu à peu d'analyses accessibles à la communauté internationale. L'une des rares analyses appréhende l'efficacité par le prix du coton syrien à l'exportation, en affirmant qu'il est inférieur à l'indice A de Cotlook de 0,07-0,08 dollars US/livre (Westlake, 2003). Par ailleurs, comme le coût de revient est largement supérieur au prix mondial, en raison d'un prix d'achat élevé aux producteurs, l'exportation se fait à perte. Quand le prix mondial est particulièrement bas, il est estimé que le différentiel peut approcher les $50 \%$ du prix mondial, induisant un déficit financier d'autant plus colossal que le surplus à exporter est important en raison d'une faible demande de l'industrie textile locale taxée dans son approvisionnement (Westlake, 2003).

L'observation de la décote de prix du coton syrien est assez étonnante car c'est un coton de qualité meilleure que celui qui sert de référence à l'indice $\mathrm{A}$. Une telle décote n'est d'ailleurs pas discernable à travers les cotations de Cotlook (ICAC, 2008). Ces cotations ne montrent pas non plus de prime pour compenser la qualité du coton syrien. On peut donc penser que l'exportation du coton syrien n'est pas suffisamment efficace pour le valoriser à sa juste valeur.

Pour autant, l'exportation à perte ne suffit pas pour porter un jugement définitif sur l'efficacité de la politique cotonnière en Syrie. La politique de soutien au prix a des effets sur les revenus directs et indirects dans les villes et campagnes qu'on ne peut occulter. Par ailleurs, à l'aune des objectifs fixés à la filière, l'efficacité de la politique appliquée ne peut être niée.

\section{Politique efficace pour atteindre les objectifs fixés}

Une progression notable de la production et du rendement

L'augmentation de la production en un peu plus d'un demi-siècle est remarquable (figure 2). Cette augmentation n'a pas été régulière. La progression dans la période 1947-1965 (avec une croissance annuelle de 17,8 \%) a été interrompue par une période de stagnation (1966-1984) résultant d'une baisse substantielle de la superficie, en relation avec la mise en ouvre de la réforme foncière. La progression a repris pendant toute la décennie des années 1990, à la suite de l'incitation donnée aux paysans pour exploiter l'eau souterraine. Il s'en est suivi une croissance annuelle de la production de $8,8 \%$ alors que tous les autres pays cotonniers enregistraient une production fluctuante.

L'augmentation de la production procède aussi pour beaucoup d'une progression remarquable du rendement. La Syrie fait partie des pays producteurs aux rendements les plus élevés - de l'ordre de $1400 \mathrm{~kg} / \mathrm{ha}$ de coton-fibre, soit plus du double de la moyenne mondiale (figure 2). C'est le résultat d'une augmen-

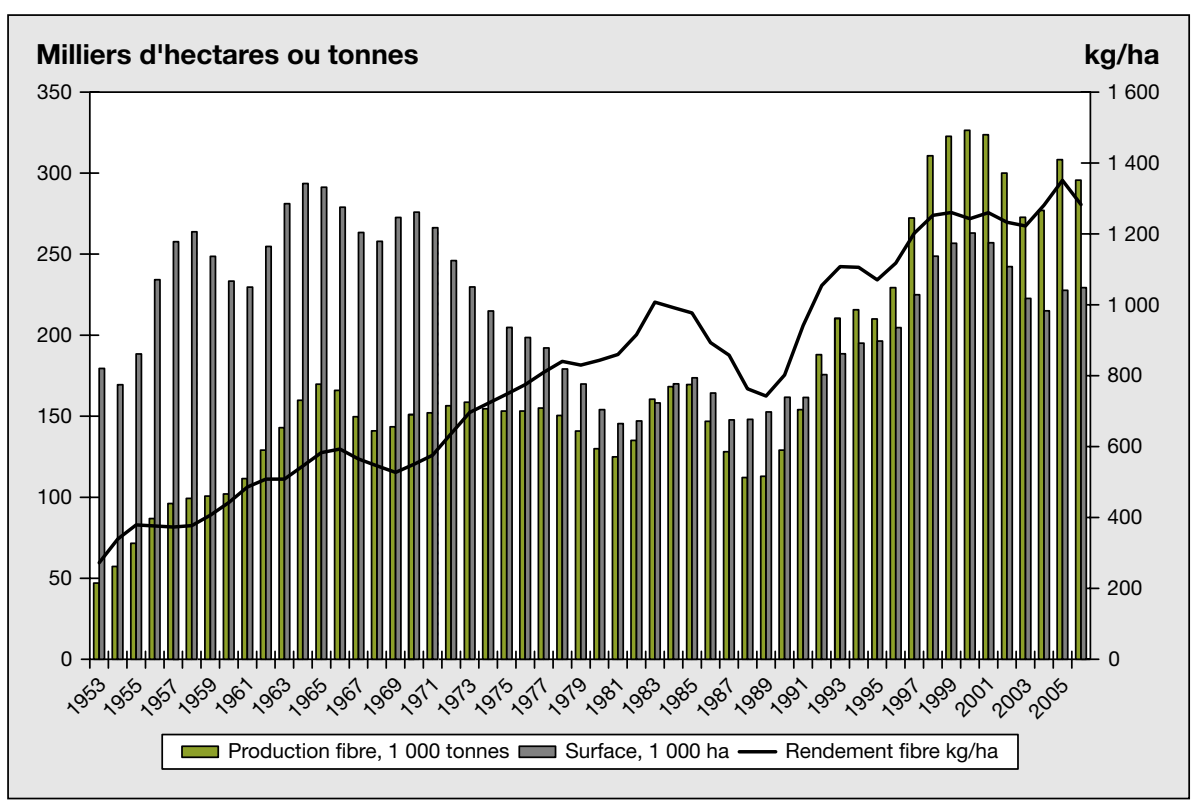

Figure 2. Évolution de la surface, production et rendement de coton-fibre, moyenne mobile sur 3 ans.

Figure 2. Evolution of cotton area and fibre production and yield, over an average of 3 years. Source : statistiques de l'International Cotton Advisory Committee (ICAC). 
tation régulière au rythme annuel de 2,5\%, à l'exception d'une baisse de rendement dans les années 1980, avant l'encouragement à l'exploitation de l'eau souterraine par les puits.

\section{Progression du rendement avec réduction du recours aux pesticides}

Les facteurs naturels, comme un ensoleillement abondant et une pression des ravageurs restreinte par la rigueur de l'hiver dans ce pays, ont certes été favorables au potentiel de rendement. L'expression de ce potentiel est néanmoins due à une recherche efficace et à une assistance technique de proximité. L'un des résultats les plus remarquables concerne la quasiélimination de l'utilisation des insecticides à travers un contrôle strict des pratiques des paysans. Depuis une dizaine d'années, les insecticides sont utilisés seulement sur quelques pour-cent des surfaces cotonnières alors qu'ils l'étaient sur plus de 25\% en 1986 (Babi et al., 1998 ; ICAC, 1998 ; ICAC, 2004). La Syrie est aussi l'un des rares pays appliquant la lutte biologique à grande échelle (ICAC, 2004) avec l'enregistrement du premier biopesticide dès 1998 (Babi et al., 1998). En conséquence, le coût du contrôle des ravageurs y est bien plus faible que dans les pays concurrents (tableau 4).

Il convient de noter qu'une nouvelle baisse de production est observée ces dernières années. C'est le résultat d'une réduction de la surface planifiée non compensée par un gain de rendement.

Un exemple de contrôle réussi de la production de coton

Depuis le début des années 2000, la prise en compte de la disponibilité en eau s'est traduite par une réduction des surfaces avec permis de culture (tableau 3) d'environ $12,5 \%$. Le soutien de l'État est ainsi modulé en fonction de la pertinence des zones géographiques pour la culture cotonnière. La politique suivie s'avère efficace car il n'y a plus de production en dehors des zones planifiées (tableau 3).

Plus grande compatibilité

de l'offre et de la demande nationale

La Syrie s'est engagée dans le développement de son industrie textile dans les années 1950 et elle y est parvenue contrairement à beaucoup de pays, par exemple d'Afrique subsaharienne. Ce fut un développement à un rythme irrégulier (figure 3). Ce développement est surtout le fait d'une forte augmentation récente, après la suppression de la taxation à l'approvisionnement.

Jusqu'à la relative libéralisation récente de l'industrie textile, le taux de croissance de cette industrie textile a été bien plus faible que celui de la production de coton. Ses besoins ont toujours été largement couverts par la production locale, laissant une grande partie disponible à l'exportation. Le surplus à exporter a avoisiné 60 \% de la production lors des dernières années, à un prix parfois très largement inférieur au prix de revient.

La cessation de la taxation de l'industrie textile a eu pour effet d'augmenter sa compétitivité et d'accroitre sa demande en coton. La gestion de l'offre a, de son côté, induit une stagnation, voire une réduction, de la production. Globalement, si les besoins intérieurs sont toujours couverts en totalité, le surplus à exporter se réduit, de même que le déficit d'une exportation à prix inférieur au coût de revient.

\section{Tableau 4. Comparaison du coût pour le contrôle des ravageurs de coton, en dollars US/ha.}

Table 4. Control cost of cotton pests in Syria and other countries, in US\$/ha.

\begin{tabular}{lcccc}
\hline Pays & $\mathbf{1 9 9 8}$ & $\mathbf{2 0 0 1}$ & $\mathbf{2 0 0 4}$ & $\mathbf{2 0 0 7}$ \\
\hline Syrie & 10 & 5 & & 5 \\
Australie (Est) & 220 & 284 & 212 & 307 \\
Turquie (Cukurova) & 370 & & 217 & 303 \\
Brésil (NO Cerrado) & 300 & 536 & 247 & 200 \\
États-Unis (national) & 141 & 145 & 138 & 156 \\
Chine & 143 & 115 & 145 & 167 \\
Cameroun & & & 9 & 39 \\
Côte d'Ivoire & & & 70 & 69 \\
Inde (Centre, pluvial) & 120 & 53 & 61 & 16 \\
\hline
\end{tabular}

Sources : enquêtes de I'International Cotton Advisory Committee (ICAC) sur les coûts en 1998, 2001, 2004 et 2007.

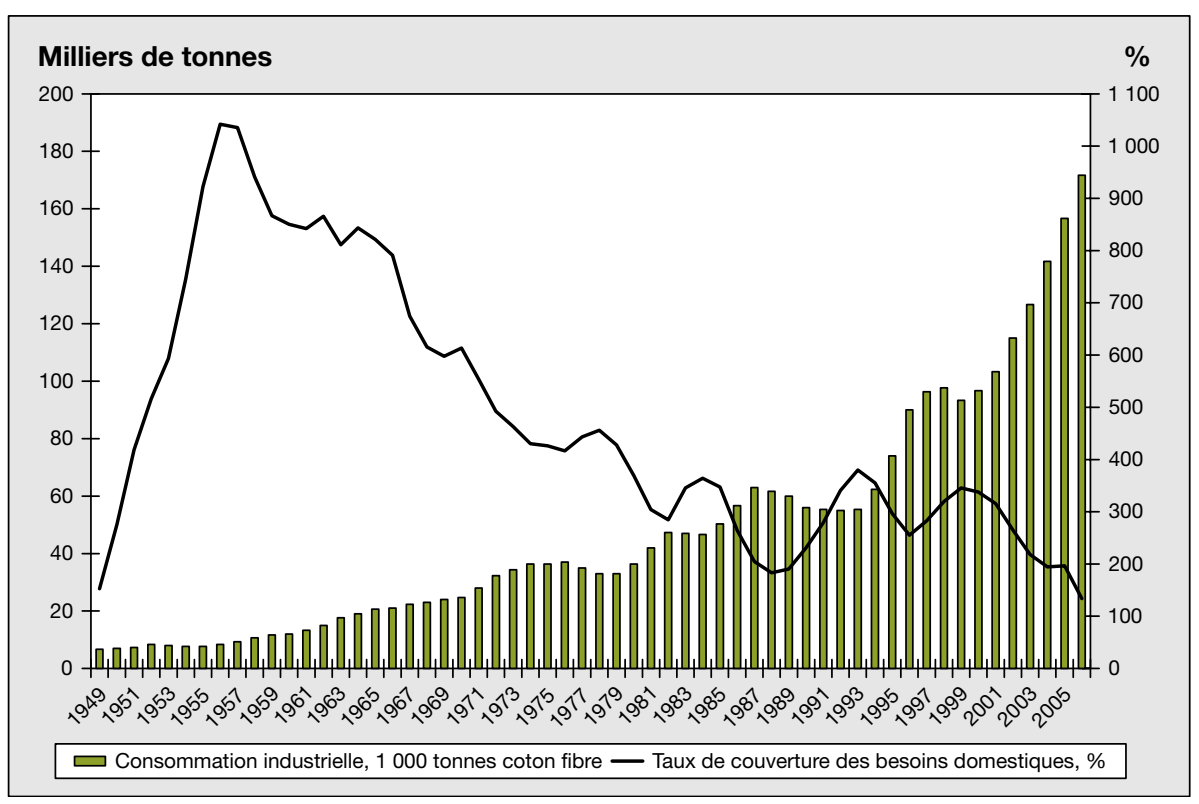

Figure 3. Évolution de la consommation industrielle du coton en Syrie et taux de couverture des besoins.

Figure 3. Evolution of mill use consumption of cotton and self-sufficiency. 


\section{Une efficacité globale difficile à évaluer et déjà menacée}

L'atteinte des objectifs témoigne d'une certaine efficacité de la politique mise en œuvre. La faiblesse du recours aux produits chimiques confère une perception positive au mode de production syrien (ICAC, 1998; ICAC, 2004). Ce pays a aussi une production substantielle de coton biologique (24 000 et 68000 tonnes en 2006 et 2007).

Les effets induits dans le domaine du soutien au revenu des populations rurales, dans la création d'emplois directs et indirects, dans l'équilibre démographique entre villes et campagnes participent à l'efficacité de la politique syrienne. Pour autant, comme déjà indiqué, l'impossibilité d'accéder aux données ne permet pas de mesurer l'ampleur de ces effets et encore moins d'apprécier l'efficience des moyens mis en ouvre pour parvenir aux résultats obtenus.

L'analyse du coût total de production (tableau 5), en comparaison des pays à rendement similaire sous irrigation (Australie), indique que l'efficacité n'est pas optimale. Comme dans beaucoup de pays, le coût de production à l'hectare en Syrie a augmenté avec le temps, bien plus que le rendement. En dépit du haut niveau de rendement, le coût unitaire du coton syrien est le plus élevé des pays considérés, que la production y soit irriguée ou pluviale, même avec des rendements bien plus bas.

Une telle appréciation est certes à pondérer par la structure de ce coût qui intègre la rémunération du travail familial et un taux de profit de $15 \%$. En comparaison des autres pays, c'est un système qui offre aux producteurs une garantie de revenu bien meilleure. L'adhésion des paysans à la production cotonnière n'est donc pas étonnante, expliquant ainsi le bon respect des surfaces planifiées.

Pour autant, les coûts de l'irrigation par pompage dans les puits et de la maind'œuvre pour la récolte sont élevés. Ils sont responsables du niveau élevé du coût total de production qui le serait encore plus si l'eau utilisée pour l'irrigation était valorisée. Cette non-valorisation de l'eau incite au mauvais usage d'une ressource rare. En moyenne, l'irrigation par gravité consomme $15000 \mathrm{~m}^{3} /$ ha (Alabdullah et Nuppenau, 2006), alors que $12000 \mathrm{~m}^{3}$ /ha suffiraient (Rida et al., 2004), aboutissant au fait que la consommation en eau pour la culture cotonnière en Syrie est la plus élevée des pays producteurs (Chapagain et al., 2005). Il y a ainsi un manque d'efficience de l'irrigation qui justifie les objectifs de la politique agricole actuelle en Syrie. Les effets de cette dernière restent cependant encore timides, l'irrigation moderne en goutteà-goutte couvre seulement $6 \%$ des surfaces irriguées. Les incitations à la moder- nisation du mode d'irrigation restent encore insuffisantes.

La prise en compte d'une gestion durable de l'eau parait aussi trop tardive et trop modérée. Les effets négatifs de l'encouragement de l'irrigation par l'eau souterraine sont bien perceptibles. Une étude conduite en 1999-2000 montre qu'une telle politique, en liaison avec le soutien au coton, a été responsable du remplacement de la production de blé et de l'orge par le coton, bien plus exigeant en eau. L'abaissement du niveau de la nappe phréatique qui en a découlé à induit la nécessité, pour les paysans, de s'endetter pour investir, non sans risque, dans le surcreusement des puits. Pour les paysans qui n'en ont pas les moyens, l'issue est d'abandonner l'agriculture et de migrer dans les villes. L'ensemble de ce processus a résulté d'une politique de subvention du gasoil pour le fonctionnement des pompes d'exhaure (Rida et al., 2004) qui n'a pas encore été vraiment revue à ce jour.

\section{Défi pour l'ajustement de la politique vis-à-vis de l'OMC}

L'adhésion à l'OMC exige la révision de la politique agricole. Cette révision est déjà préparée par des études pour identifier les filières manquant d'avantages comparatifs et pour évaluer l'évolution de cellesci dans l'hypothèse d'une réduction du

\section{Tableau 5. Comparaison entre pays du coût total et unitaire de la production de coton.}

Table 5. Total and unit production costs in Syria and other countries.

\begin{tabular}{|c|c|c|c|c|c|c|}
\hline $\begin{array}{l}\text { Mode } \\
\text { de culture }\end{array}$ & Pays & Coût total, rendement, coût unitaire & 1998 & 2001 & 2004 & 2007 \\
\hline \multirow[t]{6}{*}{ Irrigué } & Syrie & Coût total, dollars US/ha & 1804 & 2243 & & 2323 \\
\hline & & Rendement en fibre, $\mathrm{kg} / \mathrm{ha}$ & 1190 & 1224 & & 1229 \\
\hline & & Coût unitaire de la fibre, dollars US/kg & 1,52 & 1,83 & & 1,89 \\
\hline & Australie & Coût total, dollars US/ha & 1211 & 1664 & 2255 & 3260 \\
\hline & & Rendement en fibre, $\mathrm{kg} / \mathrm{ha}$ & 1532 & 1500 & 1410 & 2150 \\
\hline & & Coût unitaire de la fibre, dollars US/kg & 0,79 & 1,11 & 1,60 & 1,52 \\
\hline \multirow[t]{9}{*}{ Pluvial } & Cameroun & Coût total, dollars US/ha & & & 490 & 688 \\
\hline & & Rendement en fibre, $\mathrm{kg} / \mathrm{ha}$ & & & 492 & 379 \\
\hline & & Coût unitaire de la fibre, dollars US/kg & & & 1,00 & 1,82 \\
\hline & Côte d'Ivoire & Coût total, dollars US/ha & & & 416 & 480 \\
\hline & & Rendement en fibre, $\mathrm{kg} / \mathrm{ha}$ & & & 584 & 588 \\
\hline & & Coût unitaire de la fibre, dollars US/kg & & & 0,71 & 0,82 \\
\hline & Inde (Centre) & Coût total, dollars US/ha & 593 & 302 & 476 & 425 \\
\hline & & Rendement en fibre, $\mathrm{kg} / \mathrm{ha}$ & 325 & 272 & 327 & 350 \\
\hline & & Coût unitaire de la fibre, dollars US/kg & 1,82 & 1,11 & 1,46 & 1,21 \\
\hline
\end{tabular}

Source : calculé à partir des données des enquêtes de I'International Cotton Advisory Committee (ICAC). 
soutien de l'État. Une régression substantielle de la production cotonnière a ainsi été anticipée (Lançon et al., 2007). L’abolition ou la diminution des subventions entraîneront une réduction de la production. La récente réduction de la subvention accordée sur le gasoil, en même temps qu'une augmentation du prix des engrais, a déjà induit un emblavement en coton inférieur au plan établi (Maldonado, 2009).

La Syrie cherche à satisfaire les conditions d'adhésion à l'OMC mais elle n'a pas encore pris de décision sur les mesures jugées incompatibles avec les règles de l'OMC, comme la forte restriction de l'accès au marché syrien, le soutien des prix, la subvention à l'exportation et le rôle des agences étatiques de commercialisation (Huff, 2004). Il est possible que la Syrie étende l'application du paiement direct à l'hectare dont le niveau devra être bien raisonné pour éviter l'effondrement de sa production cotonnière et pour continuer à satisfaire les besoins de son industrie textile.

\section{Conclusion}

Le cas syrien est très singulier du fait de l'engagement fort et stable de l'État dans le développement de la production cotonnière. La force de l'engagement se traduit par la puissance du contrôle administratif tout au long de la conduite de la culture. Le système de prix resté inchangé pendant plus de 40 ans garantit des revenus attractifs aux producteurs.

La Syrie constitue un cas unique de gestion de l'offre par les permis de culture, gestion qui tient compte de la disponibilité en eau. Elle a réussi à limiter la production cotonnière aux zones où la disponibilité en eau est jugée adéquate.

$\mathrm{Au}$ regard des objectifs retenus, la politique conduite s'est révélée efficace pour augmenter la production en conformité avec la planification et pour développer l'industrie textile dont les besoins sont largement couverts. C'est le seul pays cotonnier où le recours aux insecticides est quasiment supprimé. C'est le résultat certes des investissements en recherche et en appui technique mais aussi du contexte politique du pays qui permet le contrôle strict des pratiques culturales des paysans. Pour autant, les gains restent possibles et nécessaires. La prise en compte du souci de gestion durable de l'eau reste insuffisante. La politique pour une irrigation plus économe doit être plus vigoureuse et la place du coton mérite d'être revue (Cochrane, 2008). La valorisation du coton syrien à l'exportation peut être plus conforme à sa qualité supérieure. L'évolution de la politique cotonnière doit donc se poursuivre, avec des mesures compatibles avec les règles de l'OMC tout en préservant un rôle efficace de l'État.

\section{Références}

Al-Joumani B. An assessment of possible alternative price policies for cotton in Syria. Damascus (Syria) : National Agricultural Policy Centre, 2004.

Alabdullah I, Nuppenau EA. 2006. www.tropentag.de/2006/abstracts/posters/424.pdf [consulté le 06/01/2009].

Anonyme. Étude documentaire sur le coton syrien. Damas (Syrie): Office arabe de presse et de documentation, 1972.

Anonyme. National farm data handbook Syrian Arabic Republic. New York: United Nations Organization, 1995.

Babi A, Al Salti MN, Ibrahim J. Le coton en Syrie. Proceedings "Réunion des groupes de travail du Réseau coton méditerranéen ". Montpellier (France) : Cirad, 1998.

Chapagain AK, Hoekstra AY, Savenije HHG, Gautam R. The water footprint of cotton consumption. Value of Water Research Series No. 18. Paris: UNESCO-Institute for Water Education, 2005.

Cochrane P. Syria's water shortages : agriculture, subsidies and hydropolitics. 2008. http:// backinbeirut.blogspot.com/2008/08/syriaswaterwoes-agriculture-subsidies.html [consulté le 02/06/2009].
FAO. AQUASTAT data base. 2009. www.fao. $\mathrm{org} / \mathrm{nr} /$ water/aquastat/dbase/index.stm [consulté le 11/01/2009].

Huff B. Options for reforming Syrian agricultural policy support instrument in view of WTO accession. Damascus (Syria) : National Agricultural Policy Centre and FAO project GCP/ SYR/006/ITA, 2004.

ICAC. Obtaining high yields with minimum insecticide use in Syria. ICAC Recorder 1998; XVI : 13-7.

ICAC. Zero insecticide pest control system in Syria. ICAC Recorder 2004 ; XXII : 9-14.

ICAC. World Cotton statistics. Washington (DC) : ICAC, 2008.

Khoury F. Cotton Production in Syria. In: Braud M, Campagne $\mathrm{P}$, eds. Le coton en Méditerranée et au Moyen-Orient. Actes du colloque de Montpellier, CIHEAM/ICACIIRCT. 3-7 mars 1986. Montpellier: CIHEAM/ICACIIRCT, 1988.

Lançon F, Fiorillo C, Al Ashkar H, Atiya B, Al Shareel M, Ammouri N. L'ouverture de I'agriculture syrienne à l'économie mondiale : enjeux, opportunités et défis. Cah Agric 2007; 16: 287-93. doi : 10.1684/agr.2007.0115

Macrae J. La compétitivité du coton dans le monde Pays hors zone franc. Paris: ministère de la Coopération, 1995.

Maldonado J. Syria Cotton and products annual 2009. Washington (DC) : USDA Foreign Agricultural Service, 2009.

Rida F, Aw-Hassan A, Bruggeman A. Sustainable use of groundwater in Syria. ICARDA Caravan $2004 ;(20 / 21)$ : 22-3.

Shweih R. Comparative advantages of cotton commodity chain. Damascus (Syria) : National Agricultural Policy Centre, 2006.

World Bank. The World Development Report: Agriculture for Development. Washington (DC) : World Bank, 2008.

Tschirley DL, Poulton C, Gergely N, et al. Méthode d'analyse des effets différentiels des réformes sur les filières cotonnières en Afrique. Cah Agric 2009; 18 : 385-92. doi : 10. 1684/agr.2009.0334

Westlake M. Strategic crops sub-sector. Damascus (Syria) : National Agricultural Policy Centre and FAO Project/GCP/SYR/006/ITA, 2001.

Westlake M. Syrian agriculture at the crossroads. In: Fiorillo C, Vercueil J, eds. Syrian agriculture at the crossroads. Rome: FAO, 2003. 